\title{
Transformation of ferrihydrite to goethite and the
}

\section{fate of plutonium}

Enrica Balbonit*, Kurt F. Smith' ${ }^{2}$ Liane M. Moreau ${ }^{2}$, Tian T. Li ${ }^{3}$, Melody Maloubiert, Corwin H. Booth ${ }^{2}$, Annie B. Kersting ${ }^{1}$, Mavrik Zavarin ${ }^{1}$

1 Glenn T. Seaborg Institute, Physical \& Life Sciences Directorate, Lawrence Livermore National Laboratory, P.०. Box 808, Livermore, CA 94550, USA

2 Chemical Sciences Division, Lawrence Berkeley National Laboratory, Berkeley, CA 94720, USA

3 Material Science division, Physical \& Life Sciences Directorate, Lawrence Livermore National Laboratory, Livermore, CA 94550, USA

${ }^{4}$ Department of Environmental Engineering \& Earth Sciences, Clemson University, Clemson, SC, 29634, USA ${ }^{1}$

* Email: balbonilallnl.gov; Phone: 925-422-4833. 
Supplementary information

† Currently at IJCLab (Laboratoire de Physique des des deux infinis Irène Joliot-Curie - UMR 9012) 


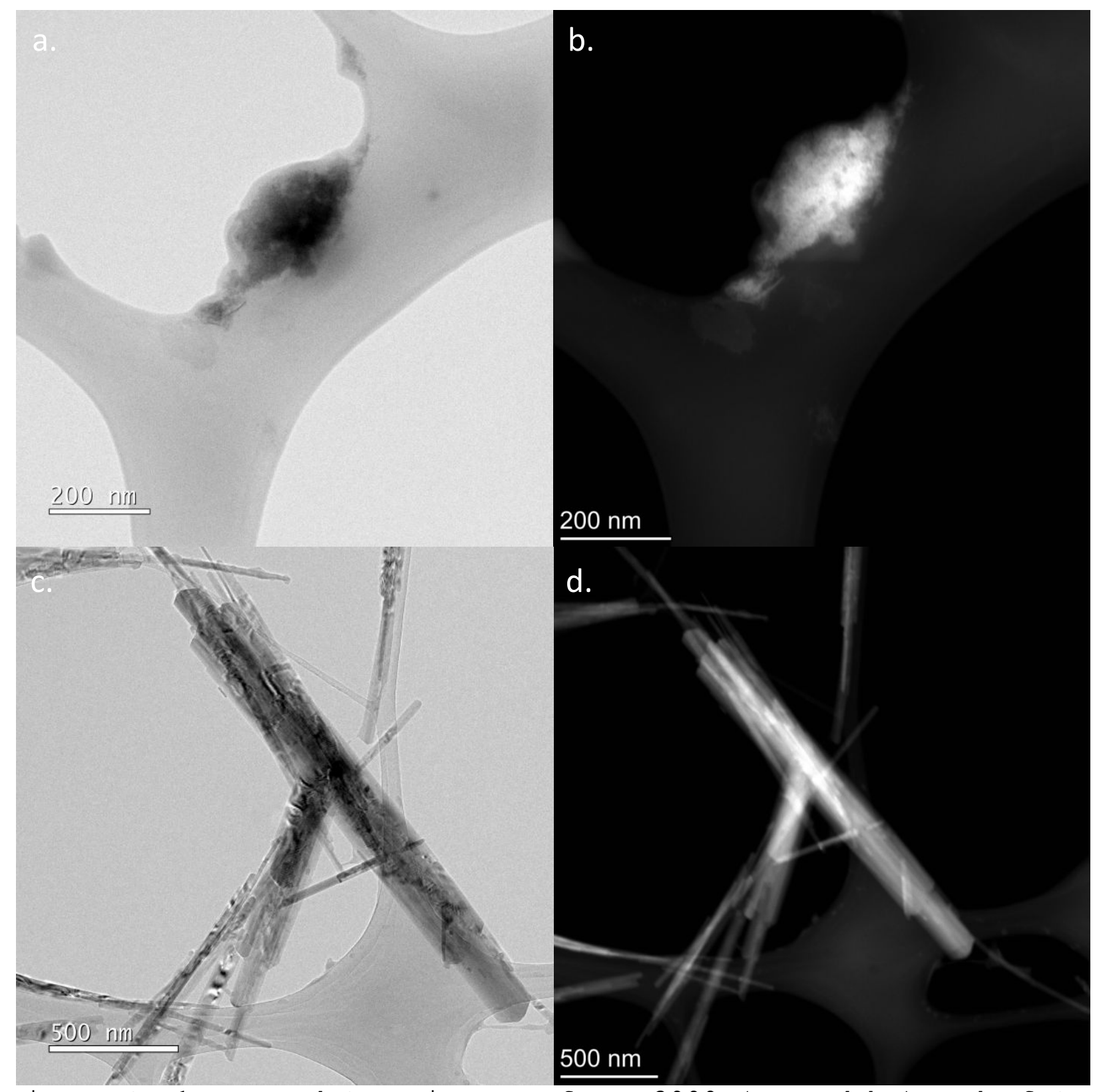

Figure SI 1 TEM and STEM images of $\mathrm{FH}_{\mathrm{C}}-3000$ (a. and b.) and of G$\mathrm{FH}_{\mathrm{C}}-3000$ (c. d. ) 


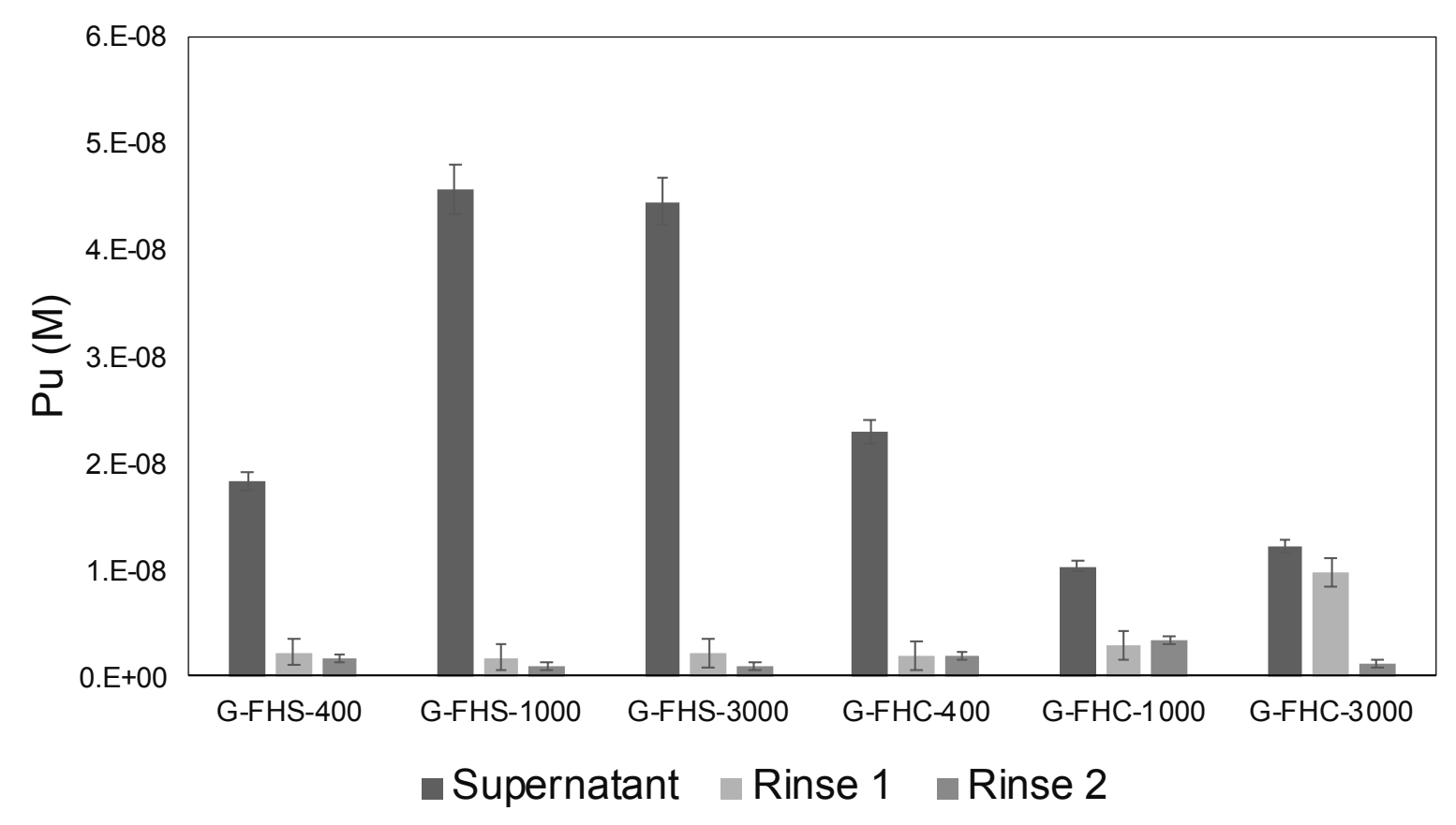

Figure SI 2: Concentration of Pu measured via liquid scintillation counting for supernatant, first and second rinse. Data shown with $2 \sigma$ error bars. The percentage of $\mathrm{Pu}$ remaining in the supernatant is $<0.2 \pm 0.08 \%$ for $\mathrm{G}-\mathrm{FH}_{\mathrm{S}}-1000$, G-FH $\mathrm{H}_{\mathrm{S}}-3000$, G$\mathrm{FH}_{\mathrm{C}}-1000$, and $\mathrm{G}-\mathrm{FH}_{\mathrm{C}}-3000$, whereas for $\mathrm{G}-\mathrm{FH}_{\mathrm{S}}-400$ and $\mathrm{G}-\mathrm{FH}_{\mathrm{C}}-400$ it ranged between $0.6-0.8 \%$ 


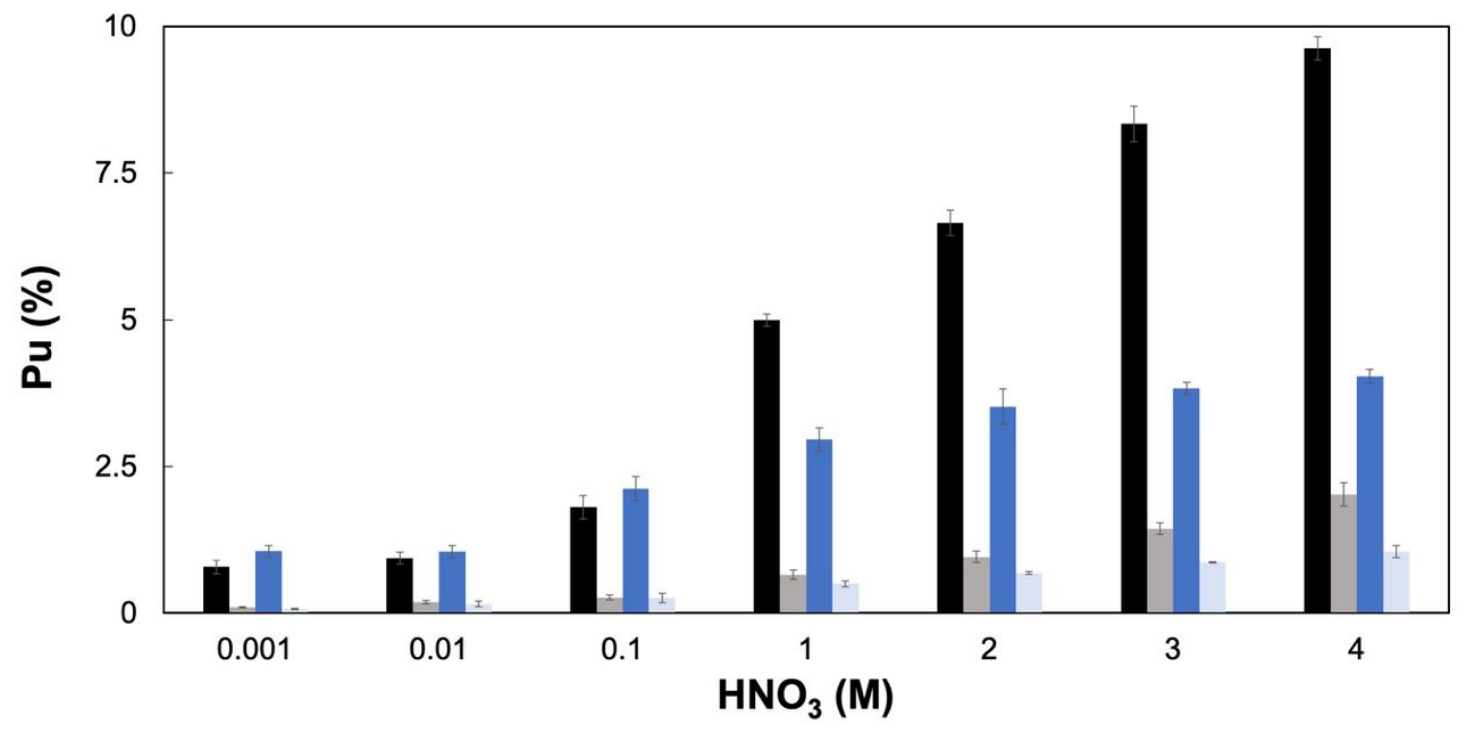

- G-FHS-3000 $=$ G-FHS-3000 (<20 $\mu \mathrm{m}) \quad$ = G-FHC-3000 $\approx$ G-FHC-3000 $(<20 \mu \mathrm{m})$

Figure SI 3: Leaching behavior of $\mathrm{Pu}$ (\%) in acidic solution (0.001-4M) for G-FH $H_{S}-3000$ (black: centrifuged supernatant; gray: filtered with $3 \mathrm{kDa}$ ultrafiltration device) and $\mathrm{G}-\mathrm{FH}_{\mathrm{C}}-3000$ (dark blu: centrifuged supernatant; light blu: filtered with $3 \mathrm{kDa}$ ultrafiltration device). Data shown with $2 \sigma$ error bars. 


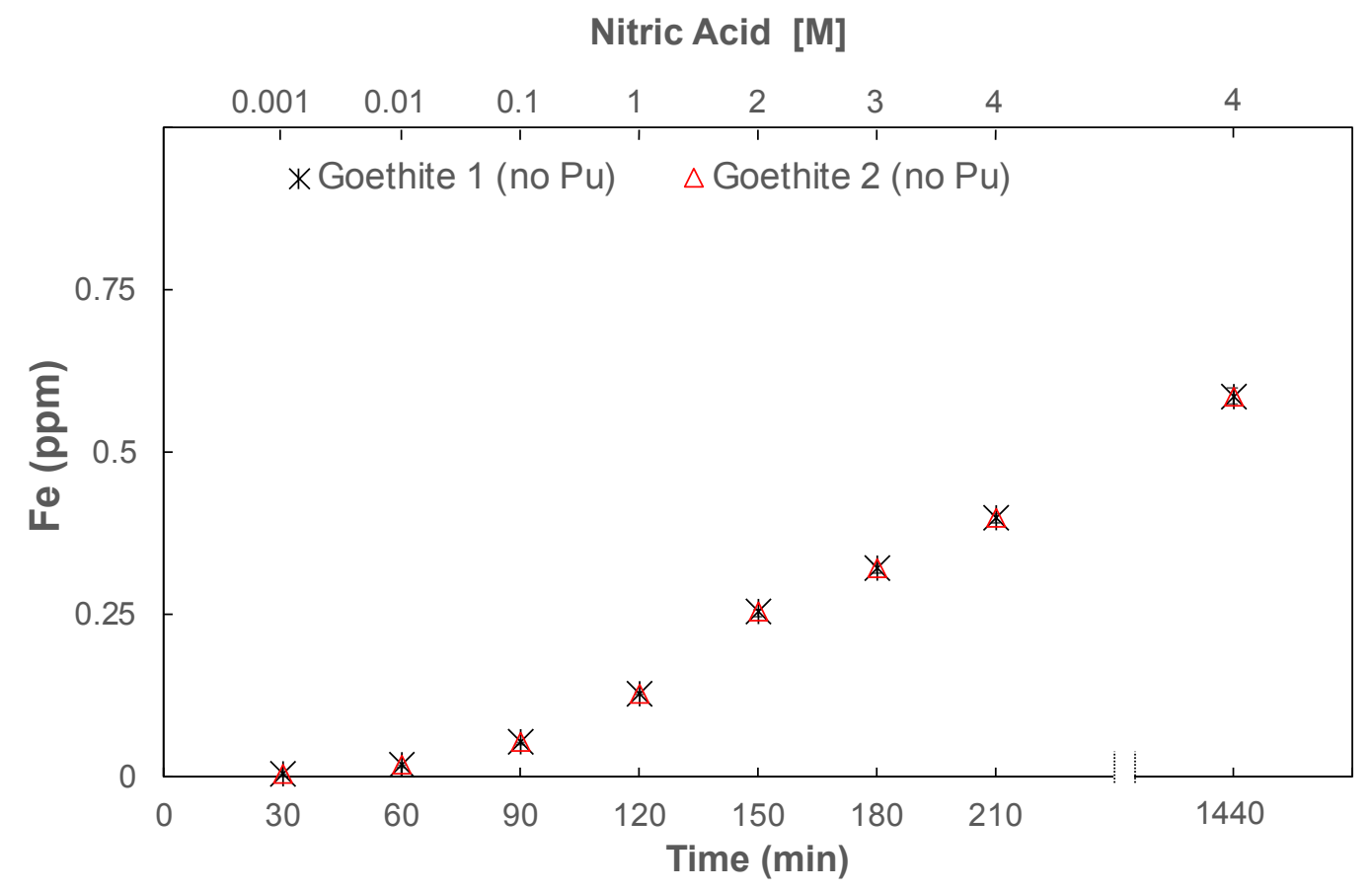

Figure SI 4 Leaching behavior of Fe in acidic solution (0.0014M) for Pu-free goethite. Data shown with $2 \sigma$ errors. 


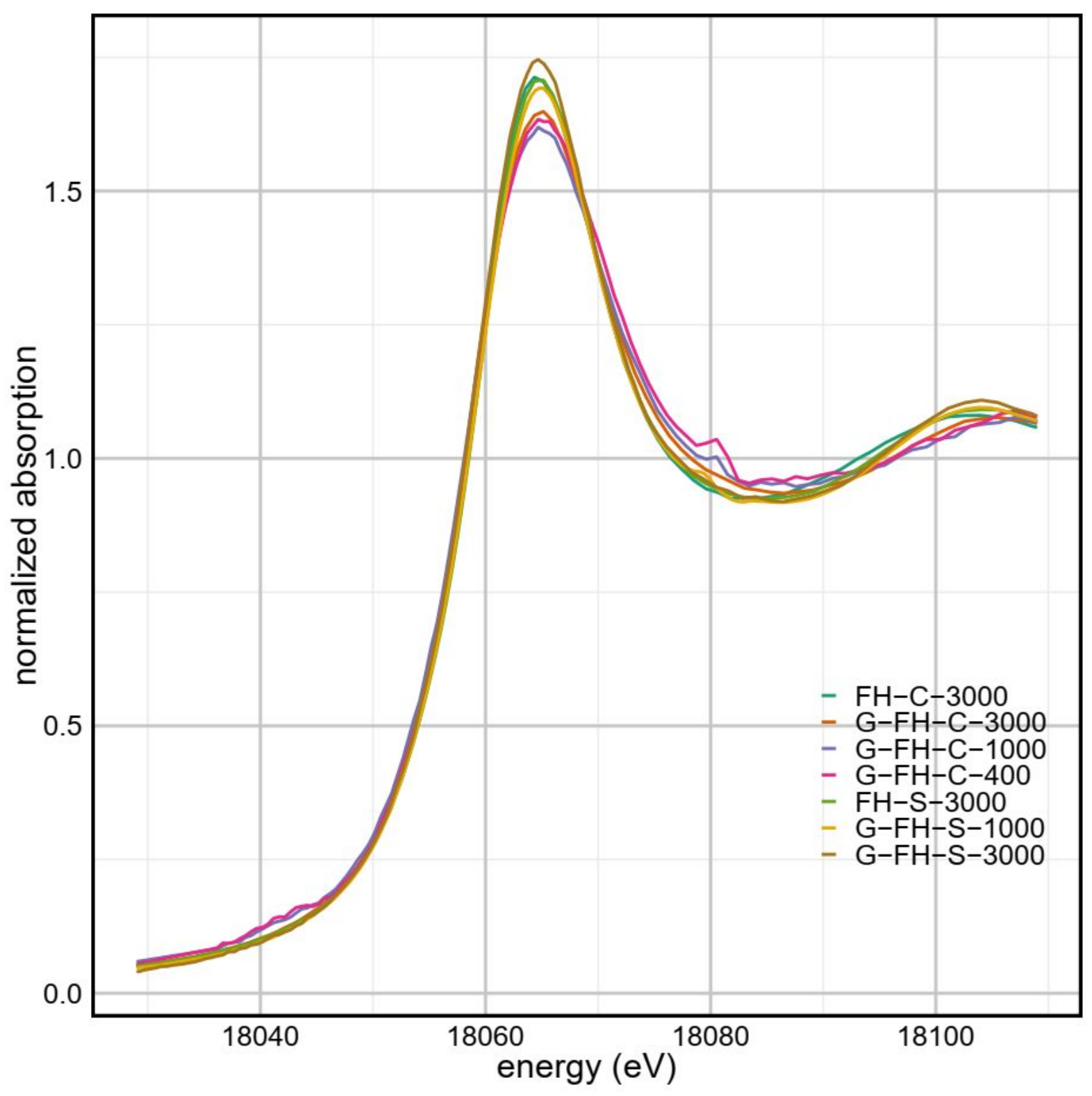

Figure SI 5. Pu L LII-edge XANES spectra collected from Puferrihydrite / Pu-goethite samples in this study. The spectra were calibrated to the Zr K edge at $17995.88 \mathrm{eV} .2$ Edge inflection points (see Table SI 1) all fall within the range of those determined by Conradson et al., $20044^{3}$ for a range of Pu(IV) solid phases confirming Pu(IV) as the predominant oxidation state in our samples. 


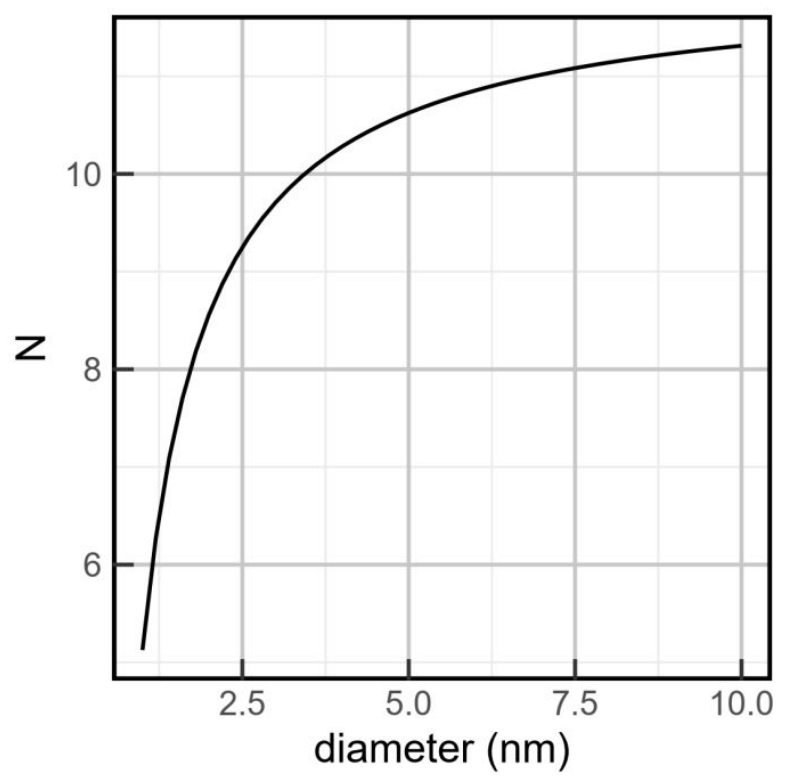

Figure SI 6 Average Pu coordination number with respect to particle size in $\mathrm{PuO}_{2}$ solids as calculated using Calvin analysis. 4

Table SI 1. Edge inflection point

(edge energy, $E_{0}$ ) of Pu-ferrihydrite / Pu-goethite samples in this study .

\begin{tabular}{cc}
\hline & \\
\hline Sample & $\mathbf{E}_{0}(\mathbf{e V})$ \\
\hline $\mathrm{G}-\mathrm{FH}-\mathrm{S}-3000$ & 18059.5 \\
$\mathrm{G}-\mathrm{FH}-\mathrm{S}-\mathrm{H}-\mathrm{S}-3000$ & 18060.8 \\
$\mathrm{G}-\mathrm{FH}-\mathrm{C}-400$ & 18060.0 \\
$\mathrm{G}-\mathrm{FH}-\mathrm{C}-1000$ & 18059.9 \\
$\mathrm{G}-\mathrm{FH}-\mathrm{C}-3000$ & 18059.6 \\
$\mathrm{FH}-\mathrm{C}-3000$ & 18059.4 \\
\hline
\end{tabular}




$\mathrm{PuO}_{2}{ }^{*}$
*from Conradson et al., $2003^{3}$ and
adjusted to a
Zr foil $\mathrm{K}$ edge of $17996 \mathrm{eV}$.

Table SI 2. Alternative EXAFS fits for FH-S-3000

\begin{tabular}{clccccc}
\hline $\begin{array}{c}\text { Fit } \\
\text { Name }\end{array}$ & Shell & $\mathbf{N}$ & $\mathbf{R}(\AA)$ & $\boldsymbol{\sigma}^{2}\left(\AA^{2}\right)$ & $\Delta \mathbf{E}_{\mathbf{0}}(\mathbf{e V})$ & $\boldsymbol{R}(\mathbf{\%})$ \\
\hline Split Fe & $\mathrm{Pu}-\mathrm{O}$ & $8(1)$ & $2.31(1)$ & $0.014(1)$ & & \\
& $\mathrm{Pu}-\mathrm{Fe}_{1}$ & $1.0(5)$ & $3.28(5)$ & $0.008^{*}$ & $-10.9(8)$ & 8.93 \\
& $\mathrm{Pu}-\mathrm{Fe}_{2}$ & $1.9(5)$ & $3.44(3)$ & $0.008^{*}$ & & \\
& $\mathrm{Pu}-\mathrm{Pu}$ & $3(1)$ & $3.80(1)$ & $0.007(2)$ & & \\
\hline Fixed N & $\mathrm{Pu}-\mathrm{O}$ & $8(1)$ & $2.32(1)$ & $0.014(1)$ & & \\
& $\mathrm{Pu}-\mathrm{Fe}$ & $3 *$ & $3.39(1)$ & $0.015(2)$ & $-10.9(8)$ & 8.91 \\
& $\mathrm{Pu}-\mathrm{Pu}$ & $3(1)$ & $3.79(1)$ & $0.007(2)$ & & \\
\hline
\end{tabular}

*indicates a fixed parameter. $\mathrm{N}$ represents the coordination number assuming an amplitude reduction factor of $1 \quad\left({ }^{5-7}\right) ; \mathrm{R}$ denotes the interatomic distance; $\sigma^{2}$ represents the Debye Waller factor; $\Delta \mathrm{E}_{0}$ represents the energy shift from the calculated energy Fermi level.

1. Neck, V.; Altmaier, M.; Seibert, A.; Yun, J. I.;

Marquardt, C. M.; Fanghaenel, T., Solubility and redox reactions of Pu(IV) hydrous oxide: Evidence for the formation of PuO2+x(s, hyd). Radiochimica Acta 2007, 95 (4), 193-207.

2. Kraft, S.; Stumpel, J.; Becker, P.; Kuetgens, U., High resolution $\mathrm{x}$-ray absorption spectroscopy with absolute energy Calibration for the determination of absorption edge energies. Review of Scientific Instruments 1996, 67 (3), 681-687.

3. Conradson, S. D.; Abney, K. D.; Begg, B. D.; Brady, E. D.; Clark, D. L.; den Auwer, C.; Ding, M.; Dorhout, P. K.; 
Espinosa-Faller, F. J.; Gordon, P. L.; Haire, R. G.; Hess, N. J.; Hess, R. F.; Keogh, D. W.; Lander, G. H.; Lupinetti, A. J.; Morales, L. A.; Neu, M. P.; Palmer, P. D.; PavietHartmann, P.; Reilly, S. D.; Runde, W. H.; Tait, C. D.; Veirs, D. K.; Wastin, F., Higher Order Speciation Effects on Plutonium L3 X-ray Absorption Near Edge Spectra. Inorganic Chemistry 2004, 43 (1), 116-131.

4. Calvin, S.; Riedel, C. J.; Carpenter, E. E.; Morrison, S. A.; Stroud, R. M.; Harris, V. G., Estimating crystallite size in polydispersed samples using EXAFS. Physica Scripta 2005, T115, 744-748.

5. Smith, K. F.; Morris, K.; Law, G. T. W.; Winstanley, E. H.; Livens, F. R.; Weatherill, J. S.; Abrahamsen-Mills, L. G.; Bryan, N. D.; Mosselmans, J. F. W.; Cibin, G.; Parry, S.; Blackham, R.; Law, K. A.; Shaw, S., Plutonium(IV) Sorption during Ferrihydrite Nanoparticle Formation. ACS Earth and Space Chemistry 2019.

6. Merroun, M. L.; Raff, J.; Rossberg, A.; Hennig, C.; Reich, T.; Selenska-Pobell, S., Complexation of uranium by cells and S-layer sheets of Bacillus sphaericus JG-A12. Applied and Environmental Microbiology 2005, 71 (9), 5532-5543.

7. Newsome, L.; Morris, K.; Lloyd, J. R., Uranium Biominerals Precipitated by an Environmental Isolate of Serratia under Anaerobic Conditions. Plos One 2015, 10 (7). 